\title{
Systemic effects of screening for retinopathy of prematurity
}

\author{
David E Laws, Clare Morton, Michael Weindling, David Clark
}

\begin{abstract}
Aims-To detect systemic complications of screening for retinopathy of prematurity (ROP), paying particular attention to the physical examination.

Methods-Oxygen saturation, pulse rate, and blood pressure were monitored before, during, and after 110 ROP screening examinations.

Results-Following topical mydriatics diastolic blood pressure was elevated by a mean of 6 (SD 7.2) $\mathrm{mm} \mathrm{Hg}$. Immediately after the examination there was a further rise in both systolic and diastolic pressure of $4.3(14.5) \mathrm{mm} \mathrm{Hg}$ and $3.3(11.6) \mathrm{mm} \mathrm{Hg}$, respectively. Oxygen saturation and pulse rate remained stable during the control period and administration of eyedrops. Saturation fell by a median of $3 \%(95 \%$ confidence interval plus or minus $1 \cdot 2 \%$ ) after the examination while there was rise in pulse rate of 7 (SD 23.1) beats per minute. This change in pulse rate was not observed in infants on concurrent methylxanthine therapy. No infant had clinically significant changes at the end of the study.

Conclusion-The initial changes in blood pressure may represent side effects of topical mydriatics but the later changes following the physical examination may be an additional response to the stress of ROP screening.

(Brf Ophthalmol 1996; 80: 425-428)
\end{abstract}

Screening for the development of acute retinopathy of prematurity (ROP) is recommended practice in the UK. ${ }^{1}$ This activity fulfils many of the requirements of a screening programme as the disease is detectable, treatable, ${ }^{2}$ and the susceptible population is identifiable $^{3}$ and accessible. ROP screening could save an estimated 50 to 100 infants from blindness in the $\mathrm{UK}^{4}$ and 320 in the USA. ${ }^{5}$ It is important, however, to ensure that in searching for treatable disease we do not expose the susceptible population to unnecessary risks from the examination itself.

Several studies have looked at the effects of mydriatic eyedrops on the preterm infant ${ }^{6-11}$ but less attention has been paid to the possible side effects of physical manipulation of the globe. It was our clinical impression that handling the infant in the course of the examination might be more disturbing than the drops alone. This hypothesis was supported by the work of Clarke et al ${ }^{12}$ who reported electrocardiographic and blood pressure changes associated with insertion of an eyelid speculum and indentation of the globe during ophthalmoscopy.

The aim of the present study was threefold. Firstly, to identify any significant systemic complications during the course of ROP screening. Secondly, to identify which part of the screening process, if any, was most likely to cause these problems. Thirdly, to determine whether there was an identifiable group of infants who were particularly vulnerable. We therefore undertook a prospective examination of the changes in pulse rate, oxygen saturation, and blood pressure during ROP screening.

\section{Patients and methods}

Infants were enrolled from the ROP screening clinics at the Liverpool Maternity Hospital and Aintree Hospitals Trust. Infants less than 32 weeks' gestation or birth weight less than $1500 \mathrm{~g}$ were included. ${ }^{1}$ Both inpatients and outpatients were examined. The outpatients were allowed 15 minutes to settle before commencement of the study.

Pupil dilatation was performed with one drop of phenylephrine $2.5 \%$ in each eye at 0 and 30 minutes. Cyclopentolate $0.5 \%$ was instilled four times at $0,15,30$, and 45 minutes. After 1 hour oxybuprocaine $0.3 \%$ anaesthetic was instilled. The examination was then performed with the infant in the supine position using a neonatal lid speculum and indirect ophthalmoscopy with a 28 dioptre lens. Each infant was examined using a standardised technique starting with the speculum being placed in the left eye. A squint hook was then placed in the conjunctival fornix to gently rotate (rather than indent) the globe medially, then laterally, to view the peripheral retina. The right eye was then examined using the same procedure. Where infants were on supplemental oxygen therapy this was continued during the examination. All examinations were performed by one observer (DC); blood pressure, pulse, and saturation readings were taken by two observers (DL, CM). Oscillometric blood pressure was recorded using a neonatal monitor (Graseby Medical, model 901 ) and the largest cuff that could comfortably be placed on the infant's thigh was used. Pulse and saturation were recorded from the wrist or foot using pulse oximetry (Ohmeda, Biox 3700e). Readings were only accepted when a satisfactory plethysmographic trace was observed.

Readings were taken according to a fixed schedule during the study period (Fig 1). Control blood pressure, saturation, and pulse recordings were taken three times over a 20 minute period before any topical therapy. 


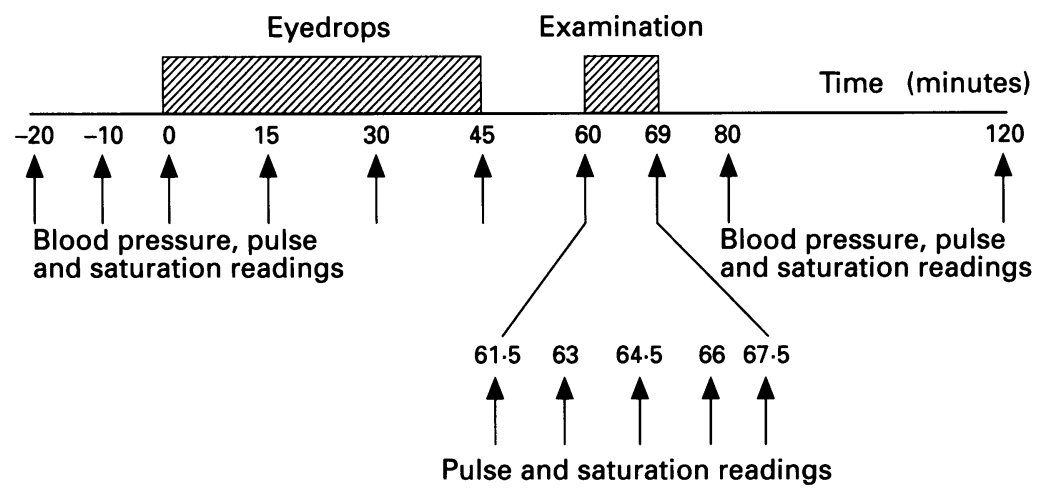

Figure 1 Schedule of examination and observations. The hatched areas show the timing of administration of the eyedrops and of the examination in minutes. The arrows indicate the time readings were taken with an expanded area showing the extra pulse and saturation readings during the examination.

A subgroup of infants had extra blood pressure, oxygen saturation, and pulse rate recordings during administration of the eyedrops to exclude any transient effects of the topical medication. ${ }^{10}$

Blood pressure, saturation, and pulse measurements were repeated on all infants immediately before the physical examination. Pulse rate and oxygen saturation were recorded during the physical examination after insertion of the lid speculum, medial, and lateral rotation of each eye to gauge the effect of each action. It was not possible to obtain blood pressure readings during the examination without interfering with its progress so these data were recorded immediately the examination finished. Follow up blood pressure, pulse, and saturation readings were taken 10 and 50 minutes after the end of the examination.

Changes in normally distributed data were analysed using the paired Student's $t$ test. Non-parametric data (for example, oxygen saturation) were analysed using the Wilcoxon signed rank test. The relation between variables such as change in blood pressure and postmenstrual age at examination were analysed using simple linear regression.

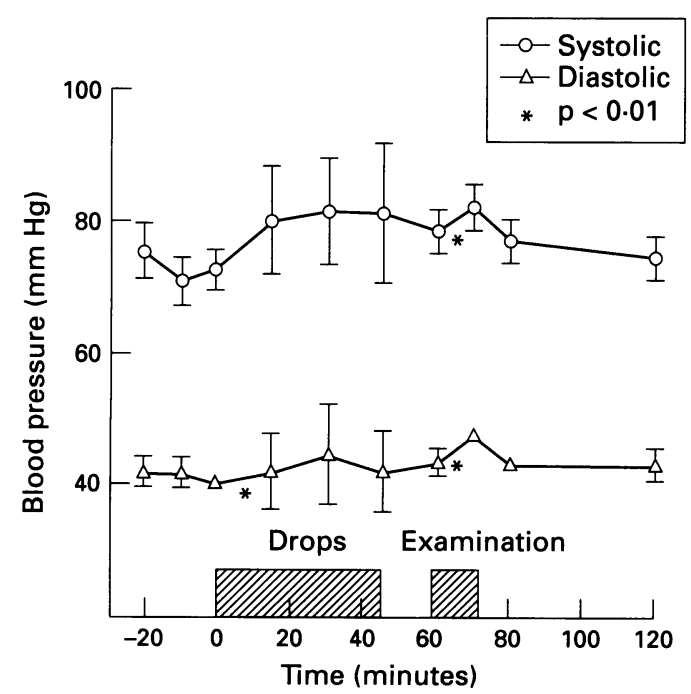

Figure 2 Mean blood pressure changes during the study with $95 \%$ confidence intervals. Asterisks placed between points indicate their statistical difference. NB: fewer recordings during administration of the eyedrops account for the wider error bars.

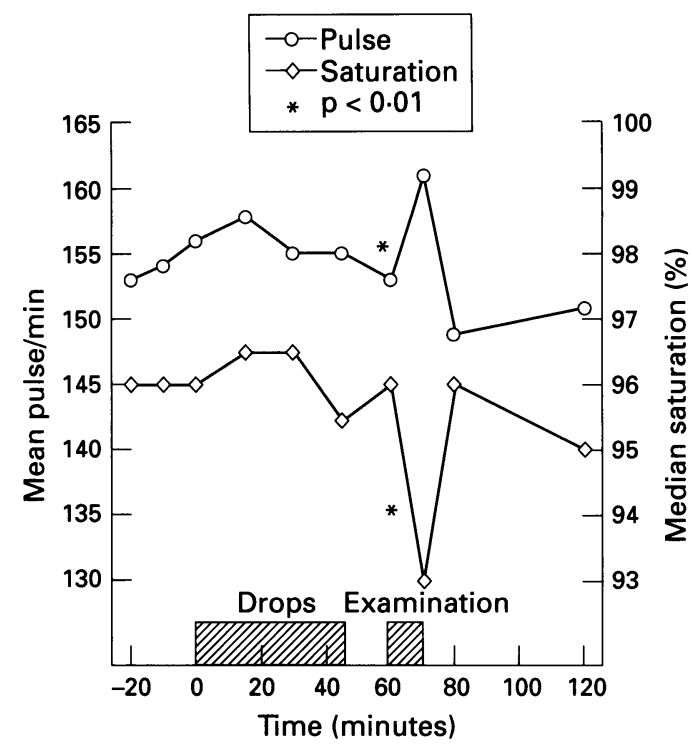

Figure 3 Mean pulse rate and median oxygen saturation showing rise in pulse and drop in saturation associated with the examination. Asterisks placed between points indicate their statistical difference.

\section{Results}

The results of 110 examinations on 56 infants (30 males and 26 females) were analysed. There was a median of two examinations per infant (range 1 to 6). The mean gestation was $27 \cdot 1$ (SD 2.4) weeks and the mean birth weight was 1003 (332) g. The mean postmenstrual age at the time of examination was $36.5(3.3)$ weeks and the mean examination lasted 8.9 minutes (partly because of the time taken to obtain satisfactory readings). In 42 of the 110 examinations infants received supplemental oxygen by nasal cannulae, face mask, or intubation.

Blood pressure changes during the course of the study are shown in Figure 2. Readings remained relatively constant during the control period with a mean systolic pressure of 73 $(17 \cdot 2) \mathrm{mm} \mathrm{Hg}$ and mean diastolic pressure of $41(11.6) \mathrm{mm} \mathrm{Hg}$ immediately before administration of the eyedrops.

Fifteen minutes after the first phenylephrine and cyclopentolate drops the cohort having extra readings taken demonstrated a statistically significant rise of diastolic pressure $(\mathrm{p}<0.01)$ but not systolic $(\mathrm{p}=0.079)$ (paired $t$ test). Both systolic and diastolic levels remained reasonably stable while the rest of the drops were administered. Immediately after the examination there was a further rise in systolic blood pressure to $82.7(18.3) \mathrm{mm} \mathrm{Hg}$ and in diastolic pressure to $47.7(11.4) \mathrm{mm}$ $\mathrm{Hg}$. Compared with the readings immediately before the examination both these changes were statistically significant $(p<0 \cdot 01)$.

Ten minutes after completion of the examination systolic and diastolic pressure had dropped to similar levels to those before the examination.

Pulse rate did not alter significantly during the control period or during administration of the eyedrops (Fig 3). Figure 4 shows the changes in pulse rate recorded during the examination. Following placement of the speculum in the left eye there was an initial drop in pulse rate from a mean of 153 beats per minute to 150 per minute which was statistically significant 


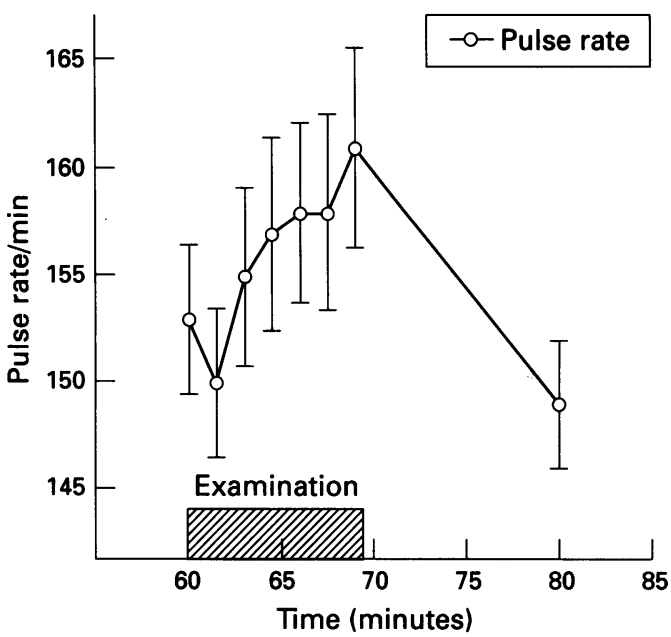

Figure 4 Mean pulse rate changes during the physical examination with $95 \%$ confidence intervals.

$(p<0 \cdot 05)$. This decrease was followed by a gradual increase, which became significant after the speculum was placed in the right eye and remained so until the end of the examination when the mean rate was 161 beats per minute $(p=0.0058)$. Ten minutes after the examination the mean pulse rate had dropped back to 149 per minute.

The median oxygen saturation was constant at $96 \%$ during the control period. During the period in which the drops were administered there was some variability but no statistically significant change. Figure 3 shows the pulse and saturation changes during the whole course of the study. There was a median drop in saturation of $3 \%$ immediately the speculum was placed into the palpebral aperture of the left eye. This lower level of oxygen saturation was maintained for the duration of the examination and was significantly different $(p<0.001)$ from the level before any physical manipulation. When handling of the infant discontinued the saturation returned to previous levels. Three infants had desaturations of over $20 \%$, one of these had desaturations before the study and one had chronic lung disease but they were otherwise in the mid range for weight, birth weight, and postmenstrual age. They recovered to previous oxygen saturation levels immediately on discontinuing the examination.

Infants receiving supplemental oxygen consistently had slightly lower oxygen saturation levels than those in air but the change in saturation in response to the examination was similar in each group. Further analysis failed to reveal any significant difference in blood pressure or pulse rate responses to the examination in infants with supplemental oxygen and in those without.

Simple linear regression did not demonstrate any obvious relation between changes in blood pressure, saturation, or pulse and the postmenstrual age at examination or weight at examination. Increased birth weight was, however, associated with a greater proportional increase in pulse rate following the examination and this effect remained after correction for postmenstrual age $(p=0 \cdot 025)$.

Data concerning current medication were also recorded in 88 cases but there was no association between change in saturation, pulse, or blood pressure and the use of antibiotics or diuretics. In 25 examinations infants were receiving concurrent treatment with a methylxanthine (theophylline or aminophylline) for apnoea of prematurity. These infants had lower birth weight and were of shorter gestation than the infants in the 63 examinations where methylxanthine therapy was recorded as not used. There were no significant differences between these two groups with regard to saturation or blood pressure. The mean pulse rate in the infants on methylxanthine was, however, consistently higher during the control period and administration of the eyedrops. During the physical examination there was no significant difference between the groups in the proportion developing an oculocardiac reflex (defined as a $10 \%$ decrease in heart ${ }^{13}$ ). A gradual rise in mean pulse rate following the initial decrease was observed for the untreated group but not for those receiving methylxanthine. Thus, the infants not on methylxanthine therapy had a greater overall rise in pulse rate related to the examination (mean 12.7 beats per minute) compared with those on treatment (mean $-3 \cdot 1$ beats per minute, $p=0.01$ ).

Parents and nursing staff were also asked to report any other problems that may have related to the examination. One infant required reventilation 18 hours after ROP screening but had developed a viral infection; one infant with previously labile blood pressure developed a hypotensive episode 8 hours after the examination but recovered without further treatment; and one infant was readmitted to another hospital the day after the examination with oesophageal reflux.

\section{Discussion}

This study demonstrates several changes that occur during the course of ROP screening.

Following systemic absorption the $\alpha$ adrenergic effect of phenylephrine raises peripheral resistance and blood pressure. This has been reported in previous studies ${ }^{11} 14$ and review articles ${ }^{15} 16$ although Isenberg et $a l^{7}$ and Bolt et al ${ }^{10}$ did not detect a rise in blood pressure using phenylephrine. The mean increase in systolic reading in this study was $5.3 \mathrm{~mm} \mathrm{Hg}$ and in diastolic $6 \mathrm{~mm} \mathrm{Hg}$ at 15 minutes, although there was a large variation in response. Lynch et al ${ }^{17}$ and Wheatcroft et al ${ }^{18}$ suggest reducing the drop size to avoid side effects, and this may be an alternative method to prevent these changes.

While there have been several studies analysing the pharmacological effects of mydriatics on the neonate few have looked in detail at the physical examination. This study has shown an additional rise in mean systolic blood pressure following the examination of $4.2 \mathrm{~mm} \mathrm{Hg}$ and in diastolic blood pressure of $3.8 \mathrm{~mm} \mathrm{Hg}$.

The pulse rate did not vary significantly during the control period or while drops were given. Using a definition of the oculocardiac 
reflex as a $10 \%$ drop in pulse rate, ${ }^{13} 24 \%$ of the infants developed the reflex at the start of the examination. Clarke et al, ${ }^{12}$ using continuous electrocardiographic recording, described this response in $13 \%$ associated with the insertion of the speculum and $31 \%$ of infants at some time during examination. Bates et al ${ }^{19}$ described an infant suffering profound bradycardia during scleral indentation but who recovered spontaneously after 2-3 minutes. The initial drop in pulse rate was followed by a gradual rise as the examination continued; one infant had a pulse rate over 200 per minute as the speculum was removed. The observed changes in pulse rate and blood pressure at the end of the examination may result from an adrenergic response to the stress of the procedure. These changes, while unlikely to be significant in terms of potential medical problems, may indicate that ROP screening is uncomfortable for the infant.

Oxygen saturation was reasonably stable in the control period and during administration of the drops but fell after placement of the speculum and remained reduced until the end of the procedure. This observation was made both in infants who cried and those who slept through the examination. No infant, including the three with greater than $20 \%$ saturation drop, suffered a clinically significant apnoeic episode after the examination stopped and saturation levels rapidly returned to earlier levels in most cases. These results contrast with the findings of Brown et al ${ }^{20}$ who looked at 80 infants undergoing peripheral retinal cryoablation for ROP, three of whom developed respiratory arrest. Batton et al ${ }^{21}$ found that five of 11 infants not previously mechanically ventilated required respiratory support after cryotherapy. The infants were of comparable weight to the infants in this study but the studies differ in the type of procedure and the time taken, the greater complication rate reflecting the greater level of intervention.

No particular age or weight group appeared to be at increased risk of raised blood pressure or decreased saturation. Heavier birthweight infants had a significantly greater percentage rise in pulse rate perhaps reflecting greater maturity of the autonomic nervous system. Infants receiving supplemental oxygen did not respond to any greater degree than their counterparts breathing air only.

There was no relation between the type of physical stimulus (that is, speculum or site of indentation) and the response in the variables measured.

Clarke et al ${ }^{12}$ in their study of the heart rate following ROP screening comment that there was no consistent relation between drug therapy, including aminophylline, and the development of the oculocardiac reflex. We were also unable to demonstrate a relation between drug therapy and the oculocardiac reflex but those on methylxanthine therapy did not have a rise in pulse rate towards the end of the examination. This observation may be due to an already increased pulse rate as a result of the therapy ${ }^{22}$ or relate to the demographic differences between the two groups.

There was no clinically significant systemic complication directly attributable to ROP screening in this study although statistically significant changes were observed. No particular infant group appeared to be at particular risk. The physical examination was related to the greatest observed response; consequently, this part of the examination should be kept as brief as possible.

1 Anonymous. ROP screening duty. College News ( $Q$ Bull $R$ Coll Ophthalmol) 1990; Autumn: 6.

2 Cryotherapy for retinopathy of prematurity cooperative group. Multicenter trial of cryotherapy for retinopathy of prematurity: preliminary results. Arch Ophthalmol 1988; 106: 471-9.

3 Fielder AR, Shaw DE, Robinson J, Ng YK. Natural history of retinopathy of prematurity: a prospective study. Eye 1992; 6: 233-42.

4 Schulenburg W, Acheson J. Cryosurgery for acute retinopathy of prematurity: factors associated with treatment success and failure. Eye 1992; 6: 215-20.

5 Javitt J, dei Cas R, Chiang YP. Cost effectiveness of screening and cryotherapy for threshold retinopathy of prematurity. Pediatrics 1993; 91: 859-66.

6 Bauer C, Trepanier Trottier M, Stern L. Systemic cyclopentolate (cyclogyl) toxicity in the newborn infant. f Pediatr 1973; 82: 501-5.

7 Isenberg S, Everett S, Parelhoff E. A comparison of mydriatic eyedrops in low-weight infants. Ophthalmology 1984; 91: 278-9.

8 Isenberg SJ, Abrams C, Hyman PE. Effects of cyclopentolate eyedrops on gastric secretory function in pre-term infants. Ophthalmology 1985; 92: 698-700.

9 Lahdes K, Huupponen R, Kaila T, Ali MT, Salminen L, Saari M. Systemic absorption of ocular cyclopentolate in Saari M. Systemic absorption of ocular cycl
children. Ger $\mathcal{F}$ Ophthalmol 1992; 1: 16-8.

10 Bolt B, Benz B, Koerner F, Bossi E. A mydriatic eye-drop combination without systemic effects for premature infants: a prospective double-blind study. $f$ Pediatr Ophthalmol Strabismus 1992; 29: 157-62.

11 Lees BJ, Cabal LA. Increased blood pressure following pupillary dilation with $2.5 \%$ phenylephrine hydrochloride in preterm infants. Pediatrics 1981; 68: 231-4.

12 Clarke WN, Hodges E, Noel LP, Roberts D, Coneys M. The oculocardiac reflex during ophalmoscopy in premature infants. Am $\mathcal{F}$ Ophthalmol 1985; 99: 649-51.

13 Dewar K, Wishart HY. The oculocardiac reflex. Proc $R$ Soc Med 1976; 69: 373.

14 Borromeo-McGrail V, Bordiuk JM, Keitel H. Systemic hypertension following ocular administration of $10 \%$ phenelyephrine eye drops in the neonate. Pediatrics 1973; 51: $1032-6$

15 Palmer EA. How safe are ocular drugs in pediatrics? Ophthalmology 1986; 93: 1038-40.

16 Pavan-Langston D, Dunkel EC. Handbook of ocular therapy and ocular side effects of systemic drugs. 1st ed. Boston: Little, Brown, 1991: 226-33.

17 Lynch MG, Brown RH, Goode SM, Schoenwald RD, Chien D. Reduction of phenylephrine drop size in infants achieves equal dilation with decreased systemic absorpachieves equal dilation with decreased syste
tion. Arch Ophthalmol 1987; 105: 1364-5.

18 Wheatcroft S, Sharma A, McAllister J. Reduction in mydriatic drop size in premature infants. $\mathrm{Br} \mathcal{F}$ Ophthalmol 1993; 77: 364-5.

19 Bates J, Burnsteine RA. Consequences of retinopathy of prematurity examinations. Case report. Arch Ophthalmol 1987; 105: 618-9.

20 Brown GC, Tasman WS, Naidoff $M$, Schaffer DB, Quinn G, Bhutani V. Systemic complications associated with retinal cryoablation for retinopathy of prematurity. Ophthalmology 1990; 97: 855-8.

21 Batton D, Ivery $\mathrm{P}$, Trese M. Respiratory complications associated with cryotherapy in premature infants. $A m \mathcal{F}$ Perinatol 1992; 9: 296-8.

22 Boobis AR, Burley D, Davies DM. Theophylline. In: Dollery C, ed. Therapeutic drugs. 1st ed. Edinburgh: Churchill Livingstone, 1991; 2: T33-41. 